\title{
Salud mental y aborto terapéutico
}

\author{
Mental health and therapeutic abortion
}

\author{
Marta B, Rondón ${ }^{1,2}$, M.D., M.S. \\ 1 Universidad Peruana Cayetano Heredia, Departamento de Clinica Médica, Unidad de Psiquiatría y Salud Mental. \\ ${ }^{2}$ Past Presidenta de la Asociación Internacional de Salud Mental para las Mujeres (IAWMH).
}

\begin{abstract}
Resumen
Se revisa el concepto de salud para argumentar que el componente mental es inherente al bienestar integral, estando la salud mental y la física intimamente interrelacionadas. Se describe la relación entre depresión y eventos del ciclo reproductivo, especialmente en cuanto al riesgo que supone el embarazo no deseado, que es un factor de riesgo para depresión posparto reportado en investigaciones realizadas en diversos lugares del mundo. Siendo así, las mujeres que presentan factores de riesgo para depresión (historia de padecimiento depresivo previo, antecedentes de adversidad o trauma en la infancia o exposición a la violencia, la historia familiar de trastorno afectivo, pobreza y falta de soporte social, enfermedades físicas concomitantes, y la mala salud del feto) deben recibir la información suficiente que les permita decidir si desean interrumpir la gestación por indicación terapéutica para proteger su salud mental. Se ofrecen recomendaciones prácticas para el reconocimiento y manejo de estas mujeres.
\end{abstract}

Palabras clave. Salud mental; Embarazo no Deseado; Depresión.

\section{Abstract}

The concept of health is reviewed to argue that the mental component as inherent to the integral wellbeing, since mental and physical health are closely related. The relationship between depression and events of the reproductive cycle is described, especially concerning the risk posed by unwanted pregnancy, a risk factor for postpartum depression as reported in studies conducted in various parts of the world. Consequently, women with depression risk factors (history of previous depressive ailment, background of infant adversity or trauma or exposition to violence, family history of affective disorder, poverty and lack of social support, concomitant physical ailments, and altered fetus health) should receive information to allow them to decide if they wish to interrupt pregnancy for therapeutic reasons in order to protect their mental health. Practical recommendations to recognize and manage these women are presented.

Keywords. Mental Health; Pregnancy, Unwanted; Depression.

An Fac med. 2015;76(4):407-11 / http://dx.doi.org/10.15381/anales.v76i4.11411

\section{INTRODUCCIÓN}

La definición de salud consagrada por la OMS (un estado de bienestar físico, psíquico y social y no la mera ausencia de enfermedad) integra los aspectos biológicos, sicológicos y sociales del bienestar y permite afirmar, como lo hace la Convención para la Eliminación de todas las Formas de Discriminación contra las Mujeres, que las mujeres tienen el derecho a gozar del más alto nivel disponible de salud física y mental. En ese sentido, la protección de la salud de las mujeres proporcionada por los instrumentos que garantizan el acceso al aborto por indicación terapéutica debe incluir la protección de la salud mental.

La OMS ha definido la salud mental como el estado de bienestar que permite a cada individuo realizar su potencial, enfrentarse a las dificultades usuales de la vida, trabajar productiva y fructíferamente y contribuir con su comunidad ${ }^{(1)}$.

Este concepto tiene, por cierto, un propósito didáctico para facilitar la capacitación y los procesos de prestación de servicios, al hacer referencia a una dimensión de la salud que no se puede separar de las otras dimensiones del bienestar. Los procesos mentales se originan en el cerebro, y la integralidad de estos depende del buen funcionamiento de los órganos del cuerpo. Así por ejemplo, en la uremia, se producen manifestaciones de delirium. Del mismo modo, las alteraciones de las emociones, como la ansiedad, producen modificaciones de variables fisiológicas como el pulso o la presión arterial, que eventualmente pueden llevar a enfermedades cardiovasculares.

La protección de la salud mental, por ende, es indispensable para asegurar un adecuado nivel de bienestar que permita a la persona vivir con dignidad.

\section{LA RESPUESTA FRENTE AL ESTRÉS Y LA AFECTACIÓN DE LA SALUD}

La disponibilidad de imágenes cerebrales cada vez más sofisticadas y precisas permite conocer los cambios estructurales y funcionales que se producen en las antiguamente llamadas 'enfermedades funcionales mentales', como la depresión ${ }^{(2)}$. La tomografía por emisión de positrones (PET) permite ver la disminución de la actividad en el lóbulo 
prefrontal de las personas afectadas por depresión y, aún más, la normalización de este parámetro luego de un tratamiento exitoso, ya sea farmacológico o psicoterapéutico. De hecho, actualmente sabemos que si la persona deprimida presenta hiperactividad metabólica de la región insular anterior derecha (que se mide mediante PET con fluorodeoxiglucosa) ${ }^{(3)}$ resulta mejor tratarla con antidepresivos que con terapia cognitiva conductual y lo opuesto ocurre cuando hay hipoactividad en esta región.

Los estresores medioambientales producen cambios en el funcionamiento y aún en la estructura cerebral, a través de cambios en el eje hipotálamohipófisis-adrenal, siguiendo las pautas descritas inicialmente por Selye, donde el cortisol se incrementa durante el estrés para permitir la respuesta adaptativa. La exposición a estrés abrumador (como ocurre en una violación sexual) o crónico y repetitivo (por ejemplo, en la violencia de compañero íntimo) puede llevar a pérdida del mecanismo de retroalimentación negativa del cortisol, dando lugar a hipercortisolemia (reportada en los trastornos depresivos de pacientes mujeres) o hipocortisolemia (descrita en el trastorno de estrés post traumático-TEPT) ${ }^{(4)}$.

A su vez, estos cambios crónicos del funcionamiento suprarrenal conllevan alteraciones del metabolismo de la glucosa, distribución anómala del tejido adiposo, cambios en la calcemia y otras que afectan integralmente a la salud.

En el sistema nervioso central (SNC), las consecuencias del estrés abrumador o repetitivo se manifiestan en ansiedad, depresión, trastorno de estrés postraumático, cambios del funcionamiento cognoscitivo y dificultades de la regulación de los impulsos. Se ha documentado muerte de células con disminución del volumen de la amígdala en mujeres con historia de abuso sexual, así como otras manifestaciones neurobiológicas de la exposición a experiencias adversas en la infancia que persisten a lo largo de la vida ${ }^{(5)} \mathrm{y}$ constituyen el sustrato biológico de los cambios de las emociones, la conducta y los procesos intelectuales (incluyendo el pensamiento) que resultan de la exposición al estrés.

Los grandes estudios epidemiológicos de salud y enfermedad mental emprendidos a partir de la década de los ochenta han permitido identificar que algunos trastornos mentales tienen un fuerte componente genético, con defectos irreversibles del neurodesarrollo, por ejemplo la esquizofrenia, y se presentan con la misma frecuencia en hombres y mujeres.

Otros, como la depresión, el alcoholismo y la psicopatía dependen en gran parte de la exposición a estresores ambientales y sus tasas de prevalencia se diferencian por sexo; así la depresión es el doble de frecuente en las mujeres que en los varones, pero el alcoholismo es hasta cuatro veces más frecuente en varones. Sin embargo estas tasas están variando, a medida que cambian los roles tradicionales de género ${ }^{(6)}$, de modo tal que en sociedades que han alcanzado mayor equidad las mujeres presentan tasas menores de depresión. De este hallazgo reportado en un análisis secundario de la Encuesta Mundial de Salud Mental con más de 170 mil sujetos, se concluye que la inequidad de género expresada en la tradicionalidad del rol femenino -exclusión del empleo remunerado, menores niveles de educación, matrimonio a edad temprana y escaso uso de anticonceptivos modernos- es un estresor para las mujeres y por ende constituye un factor de riesgo para depresión.

\section{EL EMBARAZO NO DESEADO, UN FACTOR DE RIESGO PARA DEPRESIÓN}

Los eventos relacionados con el ciclo reproductivo representan una carga importante para la salud de las mujeres, y se ha documentado extensamente los complejos factores (económicos, sociodemográficos, la salud previa, la disponibilidad y acceso de servicios asociados) con el elevado riesgo de morbilidad y mortalidad que estos conllevan ${ }^{(7)}$. Fisher señala que la falta de libertad para elegir la maternidad, el aborto inseguro, las enfermedades de transmisión sexual, la infertilidad, las pérdidas de un embarazo, los partos prematuros, la fístula genital causan sufrimiento y desazón en las mujeres, que eventualmente pueden manifestarse como ansiedad, depresión u otros trastornos ${ }^{(8)}$.

La depresión, que afecta en los países de bajos y medianos ingresos entre 15 a $40 \%$ de mujeres embarazadas, es un trastorno mental con base neurobiológica, incapacitante, de curso recidivante, que se acompaña de otras condiciones físicas y mentales y que puede dar lugar a la muerte por suicidio. Interfiere significativamente con el funcionamiento adaptativo de las personas afectadas y disminuye, en el caso de mujeres jóvenes, las posibilidades de realizar plenamente el potencial personal, en lo académico y lo laboral ${ }^{(9)}$.

La depresión perinatal (en la gestación y hasta doce meses posparto) tiene consecuencias severas para la mujer y el producto. Se ha documentado que las mujeres deprimidas presentan un riesgo incrementado de complicaciones obstétricas ${ }^{(10)}$, tales como cesárea, partos instrumentados, necesidad de analgesia epidural y mayor número de admisiones a unidades de cuidados intensivos, así como de prematuridad, retardo del crecimiento intrauterino y microcefalia. En el primer año de vida, los hijos de mujeres deprimidas tienen mayor riesgo de que se retrase la lactancia, no se cumplan los esquemas de vacunación, mayor frecuencia de episodios diarreicos y, por ende, desnutrición; además, existen reportes de retardo del desarrollo motor y social ${ }^{(11)}$.

El suicidio es poco frecuente en la gestación, pero aun así, en países donde se ha logrado controlar las causas prevenibles de muerte, es una de las primeras causas de mortalidad materna ${ }^{(12)}$. A lo largo de los años, los investigadores han encontrado que el suicidio en mu- 
jeres embarazadas se relaciona con el embarazo no deseado y con situaciones de violencia física y sexual de las que la mujer no puede escapar ${ }^{(13,14)}$.

El embarazo no deseado (figura 1) es un estresor que amenaza la homeostasis al interferir con el proyecto de vida y las expectativas de la mujer; representa un riesgo de perpetuación de la pobreza al interferir con las oportunidades de educación y trabajo y se acompaña de estigmatización y violencia. Una mujer que se encuentra en esta situación decide cómo enfrentarla. Sobre esa decisión pesan factores internos (sus valores y expectativas, su nivel educativo y el grado de autonomía que ha alcanzado) y externos (el apoyo de la pareja y el soporte social con que cuenta, la disponibilidad de información y servicios sanitarios, la legislación). La mujer con embarazo no deseado buscará adaptarse a la situación, preservando su homeostasis. Su decisión de interrumpir el embarazo o continuarlo puede dar lugar a consecuencias desfavorables a corto plazo, a trastornos mentales persistentes (depresión) o a la preservación de la salud mental, dependiendo de sus antecedentes, su estado actual de salud, el soporte social y los servicios de salud disponibles.

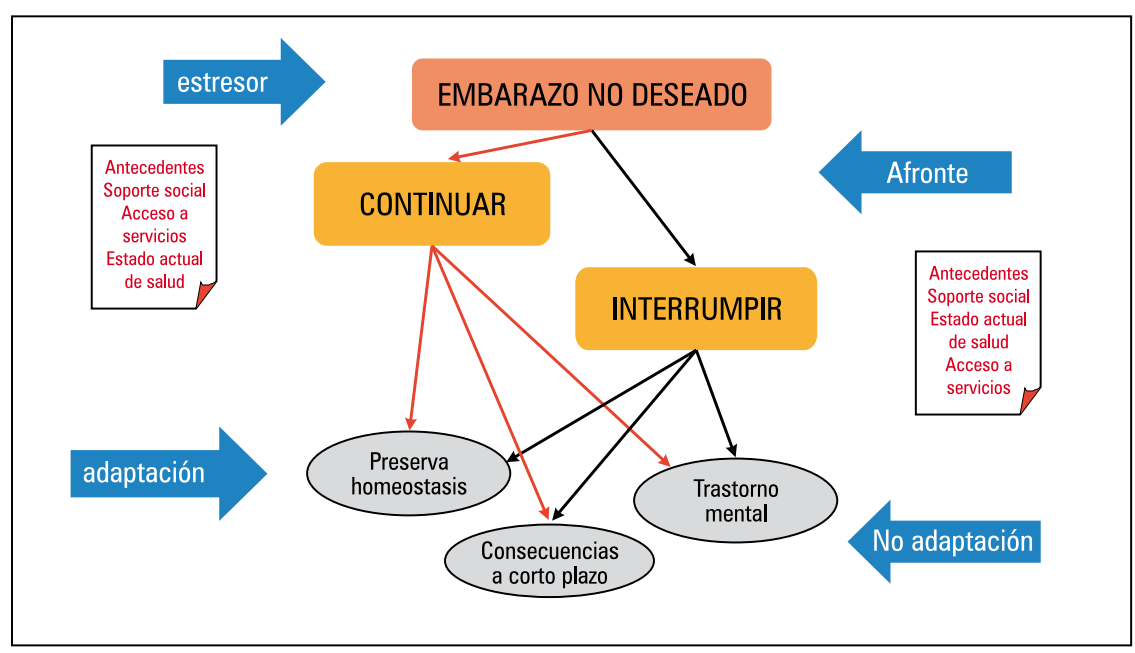

Figura 1. El embarazo no deseado es un estresor para la mujer, que puede decidir llevarlo a término o no; ambas decisiones, dependiendo de los antecedentes, el soporte social, el estado de salud actual y su acceso a servicios, pueden resultar en consecuencias desfavorables a corto y

Munk Olsen ha examinado el riesgo para la salud mental de las mujeres que abortan en comparación con aquellas que llevan el embarazo a término en una cohorte de 86420 adolescentes y mujeres que tuvieron un aborto provocado de primer trimestre y 280950 que llevaron un embarazo a término. Encontraron que el riesgo de establecer un primer contacto con un proveedor de salud mental (para consulta ambulatoria u hospitalización) de las mujeres que abortaron no aumentó significativamente durante los doce meses postaborto $(14,6$, intervalo de confianza al 95\% [IC] 13,7 a 15,6 antes del aborto y 15,2 (IC95\% 14,4 a 16,1) después del aborto). Por el contrario, las que decidieron continuar el embarazo incrementaron significativamente su necesidad de atención mental: 3,9 (IC95\% 3,7 a 4,2) antes del parto y 6,7 (IC95\% $6,4$ a 7,0$)$ posparto, $\mathrm{p}<0,001$.

Es decir, las mujeres que dan a luz tienen mayor riesgo de necesitar una atención psiquiátrica que las que no lo hacen, independientemente de la intención de tener el niño ${ }^{(15)}$.

La asociación entre embarazo no deseado y depresión posparto ha sido también investigada, partiendo de la hipótesis expuesta que representa un

$$
\text { largo plazo. }
$$

estresor para la mujer, que da lugar a síntomas de ansiedad y depresión en el embarazo que, a su vez, generan depresión posparto.

Mercier y cols (16) investigaron la trayectoria de los síntomas depresivos en 983 mujeres con embarazo planificado (433), inoportuno pero aceptado (207) y no deseado (40); encontraron que el embarazo planificado tiene menor riesgo de causar depresión y que el embarazo no deseado (no el inoportuno, sino aquel que la mujer no desea) duplica el riesgo de depresión posparto para la mujer.

Diversos estudios han encontrado la relación entre depresión posparto y gestación no deseada ${ }^{(17-19)}$. El efecto perjudicial de esta vivencia de embarazo no deseado llevado a término por condiciones del entorno persiste por muchos años ${ }^{(17)}$.

En nuestro medio, Luna Matos, Salinas y Luna han estudiado un grupo de gestantes del Instituto Materno Perinatal, encontrando que los síntomas de depresión gestacional medidos con la escala de depresión de Edinburgo son altamente prevalentes (41\%) y que el $46 \%$ de mujeres depresivas reportaron embarazo no deseado ${ }^{(18)}$.

El estrés de la gestación no deseada se manifiesta en cambios neurobiológicos, como por ejemplo la disminución del factor neurotrófico derivado del cerebro (BDNF), que es un marcador biológico de depresión. Se trata de una proteína implicada en el crecimiento neuronal, la plasticidad de las sinapsis y en la neurogénesis. Se ha encontrado que las concentraciones en sangre periférica de BDNF se encuentran reducidas en sujetos deprimidos y que se normalizan luego del tratamiento antidepresivo ${ }^{(19)}$.

En una muestra de 958 mujeres embarazadas del Instituto Nacional Materno Perinatal, Fung y cols han encontrado una asociación entre menores niveles de BDNF y depresión gestacional medida con PHQ-9, y que mujeres que reportaron embarazo no deseado presentaban BDNF bajo ${ }^{(20)}$. 
Interesa por lo tanto extender el acceso al aborto terapéutico a las mujeres con gestación no deseada que presenten otros factores de riesgo para depresión, puesto que la enfermedad depresiva es incapacitante, causa por ello detrimento para la madre y el infante y conlleva el riesgo de suicidio. Los factores de riesgo identificados para depresión perinatal son la historia de padecimiento depresivo previo, antecedentes de adversidad o trauma en la infancia, la historia familiar de trastorno afectivo, el antecedente de exposición a la violencia en todas sus formas, la pobreza y falta de soporte social, las enfermedades físicas concomitantes y la mala salud del feto.

En este sentido, es claro que el embarazo relacionado con la violencia sexual, es decir el que resulta de una violación, es un serio factor de riesgo para depresión y otros padecimientos mentales serios, como el trastorno de estrés postraumático. Por lo tanto, la mujer con este antecedente también debe acceder si lo desea a la interrupción de la gestación para proteger su salud mental.

Del mismo modo, llevar un embarazo con un feto malformado incompatible con la vida extrauterina se enmarca dentro de los factores de riesgo para depresión y la gestante con esta condición debe recibir el mismo trato.

\section{RECOMENDACIONES PRÁCTICAS}

El control prenatal proporciona una ocasión ideal para establecer una relación de confianza y respeto con la mujer que permita inquirir sobre su aceptación del embarazo, sus antecedentes personales y familiares de enfermedades mentales y su exposición a la violencia, ya sea del compañero íntimo o sexual por otro perpetrador.

Las guías de práctica clínica vigentes recomiendan la aplicación del Cuestionario de Salud Personal-9 (PHQ-9, por sus siglas en inglés) para detectar dificultades emocionales en la adaptación del embarazo. Las pacientes con punta- jes sugerentes de depresión (9 o más) y las que presenten alguno de los factores de riesgo antes anotados deben recibir información pertinente acerca de la gravedad de la enfermedad depresiva y de la posibilidad de solicitar la interrupción del embarazo no deseado.

Por otro lado, las mujeres en edad reproductiva que cursan con un trastorno mental deben recibir consejería para anticoncepción y requieren ser alentadas a usar un método seguro, para planificar su embarazo en una época en que se encuentren estables. La posibilidad de un embarazo no deseado debe ser reducida lo más posible en estas pacientes, ya que para ellas el aborto también es un estresor importante.

\section{COMENTARIO FINAL}

La salud es un bien preciado; el derecho de todas las personas al nivel más alto de salud disponible está consagrado en documentos internacionales y en la Constitución peruana. La salud mental es inherente al concepto de bienestar integral, y resulta indispensable para la vida digna y el desarrollo individual y de las comunidades ${ }^{(21)}$.

El embarazo no deseado supone una situación de estrés, ya que amenaza el proyecto de vida de la mujer y la enfrenta al riesgo de pobreza, violencia y discriminación. Por ello, produce manifestaciones depresivas y ansiosas con un sustrato neurobiológico que pueden llevar a la mujer a la depresión posparto.

La depresión es una enfermedad del cerebro que produce incapacidad, tiene un curso recidivante y conlleva riesgo de suicidio.

Ninguna mujer puede ser obligada a asumir el riesgo de un padecimiento mental que altera el funcionamiento cerebral, que tiene síntomas que alteran su calidad de vida, su funcionamiento adaptativo laboral y familiar, y que la exponen a la muerte por suicidio.

\section{REFERENCIAS BIBLIOGRÁFICAS}

1. OMS/OPS. ¿Que es la salud mental? En; http:// www.who.int/features/qa/62/es/index.html.

2. WHO \& WONCA. Integrating mental health into primary care: a global perspective. Geneva: World Health Organization. 2008.

3. McGrath CL, Kelley ME, Holtzheimer PE, Dunlop BW, Craighead WE, Franco AR, Craddock RC, Mayberg HS. Toward a neuroimaging treatment selection biomarker for major depressive disorder. JAMA Psychiatry. 2013 Aug;70(8):821-9. doi: 10.1001/jamapsychiatry.2013.143.

4. Yehuda R, Bierer LM, Schmeidler J, Aferiat DH, Breslau I, Dolan S. Low cortisol and risk for PTSD in adult offspring of holocaust survivors. Am J Psychiatry. 2000 Aug;157(8):1252-9.

5. Heim C, Plotsky PM, Nemeroff CB. Importance of studying the contributions of early adverse experience to neurobiological findings in depression. Neuropsychopharmacol. 2004 Apr;29(4):641-8.

6. Seedat S, Scott KM, Angermeyer MC, Berglund $P$, Bromet EJ, Brugha TS, et al: Cross national associations between gender and mental disorders in the WHO World Mental Health Surveys. Arch Gen Psychiatry. 2009 July;66(7):785-95. doi: 10.1001/ archgenpsychiatry.2009.36.

7. World Health Organization and United Nations Population Fund: Mental health aspects of women's reproductive health: a global review of the literature. UN and UNPFA, 2009, p IX. Disponible en http:// www.who.int/reproductivehealth/publications/ general/9789241563567/en/, última revisión 20 julio 2015 ,

8. Patel V, Oomman NM. Mental health matters too: gynaecological morbidity and depression in South Asia. Reprod Health Matters. 1999;7:30-8.

9. Berndt ER, Koran LM, Finkelstein SN, Gelenberg AJ, Kornstein SG, Miller MI, Thase ME, Trapp GA, Keller MB. Lost human capital from earlyonset chronic depression. Am J Psychiatry. 2000;157(6):940-7.

10. Chung T, Lau T, Yip A, Chiu H, Lee D. Antepartum depressive symptomatology is associated with adverse obstetric and neonatal outcomes. Psychosom Med. 2001 Sep/Oct;63(5):830-4.

11. WHO, UNPF. Maternal mental health and child health and development in low and middle-income countries: report of the meeting held in Geneva, Switzerland, 30 January - 1 February, 2008:9-10.

12. Department of Health. Report of the Confidential Enquiries into Maternal Deaths. London, Department of Health. 1999.

13. Brockington I. Suicide in women. Int Clin Psychopharmacol. 2001;16:S7-S19.

14. Frautschi S, Cerulli A, Maine D (1994) Suicide during pregnancy and its neglect as a component of maternal mortality. Int J Gynaecol Obstet. 1994;47:275-84.

15. Munk-Olsen T, Laursen TM, Pedersen CB, Lidegaard $\varnothing$, Mortensen PB.. Induced first trimester abortion and risk of mental disorder. N Engl J Med. 2011;364:332-9. doi: 10.1056/NEJMoa0905882.

16. Mercier RJ, Garrett J, Thorp J, Siega-Riz AM. Pregnancy intention and postpartum depression: secondary data analysis from a prospective cohort. BJOG. 2013 Aug;120(9):1116-22. doi: 10.1111/1471-0528.12255. Epub 2013 May 8.

17. Hook H. Refused abortion: a study of 249 women whose applications were refused by the National Board of Health in Sweden. 1963:3:156.

18. Luna Matos ML, Salinas Piélago J, Luna Figueroa A. Depresión mayor en embarazadas atendidas 
en el Instituto Nacional Materno Perinatal de Lima, Perú. Rev Panam Salud Publica. 2009;26(4):310-4.

19. Bocchio-Chiavetto $L$, Bagnardi $V$, Zanardini $R$, Molteni R, Nielsen MG, Placentino A, Giovannini C, Rillosi L, Ventriglia M, Riva MA, Gennarelli M. 2010. Serum and plasma BDNF levels in major depression: a replication study and meta-analyses. World J Biol Psychiatry. 2010;11:763-73. doi: 10.3109/15622971003611319.

20. Fung J, Gelaye B, Zhong QY, Rondon MB, Sanchez SE, Barrios YV, Hevner K, Quu C, Williams MA
Association of decreased serum brain-derived neurotrophic factor (BDNF) concentrations in early pregnancy with antepartum depression. BMC Psychiatry. 2015 Mar 10;15:43. doi: 10.1186/ s12888-015-0428-7.

21. Tribunal Constitucional Sala Primera. Sentencia recaida en el Expediente $N^{\circ} 05408-$ 2007-PHC/TC F.J.6 en el párrafo 6 del Fundamento. Disponible en: http://www.tc.gob.pe/ jurisprudencia/2008/05408-2007-HC.html, Vista por última vez el 5 septiembre 2015.
Declaración de conflicto de intereses:

Ninguno

Correspondencia:

Marta B, Rondón, M.D., M.S.

Calle José De La Torre Ugarte 471-302. Lima 12, Perú

Correo electrónico:mbrondon@gmail.com 